\title{
Combined analysis for anomalous Higgs-gauge boson couplings in $\gamma$-proton collisions at the LHC
}

\section{S. Taheri Monfared*}

School of Particles and Accelerators, Institute for Research in Fundamental Sciences (IPM), P.O. Box 19395-5531, Tehran-Iran

Department of Physics, Faculty of Basic Science, Islamic Azad University Central Tehran Branch (IAUCTB), P.O. Box 14676-86831, Tehran, Iran

E-mail: sara.taherieipm.ir

\section{Sh. Fayazbakhsh}

School of Particles and Accelerators, Institute for Research in Fundamental Sciences (IPM), P.O. Box 19395-5531, Tehran-Iran

Department of Physics, Faculty of Basic Science, Islamic Azad University Central Tehran Branch (IAUCTB), P.O. Box 14676-86831, Tehran, Iran

E-mail: shfayazbakhsh@ipm.ir

\section{Mohammadi Najafabadi}

School of Particles and Accelerators, Institute for Research in Fundamental Sciences (IPM), P.O. Box 19395-5531, Tehran-Iran

E-mail: mojtabaeipm.ir

The anomalous $H Z \gamma$ coupling is studied through the process $p p \rightarrow p \gamma p \rightarrow p H X$ at the LHC. Utilizing an effective Lagrangian with dimension six operators, new physics effects beyond the standard model are explored in this paper. The applied model includes all kinds of Higgs boson interactions in both $\mathrm{CP}$-even and CP-odd structures. The accurate constraints on anomalous $H Z \gamma$ couplings are numerically analyzed and the results corresponding to the combination of the efficient Higgs decay channels at three different forward detector acceptance regions are presented. Our numerical results propose that the Higgs photoproduction is a reliable complementary channel to study the anomalous $H Z \gamma$ vertices.

XXIV International Workshop on Deep-Inelastic Scattering and Related Subjects

11-15 April, 2016

DESY Hamburg, Germany

\footnotetext{
*Speaker.
} 


\section{Status of the Higgs-Gauge Boson Anomalous Couplings}

The standard model (SM) of particle physics is well-tested at low energy experiments. However, to explore the new physics (NP) effects on the observed phenomena which are not predicted by the SM, one has to follow non-SM models from a phenomenological point of view. In a model independent approach, an effective Lagrangian with modified interaction terms is proposed, that includes gauge invariant non-renormalizable effective operators after integrating out heavy degrees of freedom beyond $\mathrm{TeV}$ scale. There is no evidence of NP discovered at the LHC run-I, up to now, so the physicists attempt to search for signals of NP at the LHC run-II [1].

Following the discovery of the Higgs boson, the anomalous interactions of this field are also theoretically studied in the literature [2, 3]. In the SM, the estimation of the Higgs boson decay width in the $H \rightarrow Z \gamma$ channel is equal to $6 \times 10^{-6} \mathrm{GeV}$. This is computed from the Higgs particle coupling to photon and $Z$ boson through the loop contributions of $W$ boson and top quark interactions. However, the observed 95\% confidence level (C.L.) decay width for this process is about 10 times more than the predicted value by the SM [4, 5]. This discrepancy is a motivating aspect to explore NP effects through the analysis of stringent constraints on both CP-even and CP-odd anomalous $H Z \gamma$ couplings and their collider implications [6].

In the present paper, we focus on the Higgs production cross section considering the anomalous $H Z \gamma$ vertex in a single diffractive process at the LHC and one of the protons in a $p p$ collision remains intact. The fractional proton energy, $\xi$, as the detector acceptance region for detecting forward protons, is approximately equal to $\xi=E_{\gamma} / E_{\mathrm{p}}$ where, $E_{\gamma}$ and $E_{\mathrm{p}}$ are the energies of the emitted photon and the incoming proton, respectively. In what follows, the constraints on the anomalous $H Z \gamma$ couplings, at center of mass energy $\sqrt{s}=14 \mathrm{TeV}$, are discussed for three different acceptance regions, $0.0015<\xi<0.5,0.0015<\xi<0.15$, and $0.1<\xi<0.5$ of the CMS and ATLAS detectors [7].

To generalize the SM with the NP contributions, we start with an effective Lagrangian truncated at dimension six interaction terms, ignoring possible dimension five operators [1],

$$
\mathscr{L}_{\text {eff. }}=\mathscr{L}_{\mathrm{SM}}+\sum_{i} \frac{c_{i}^{(6)} \mathscr{O}_{i}^{(6)}}{\Lambda^{2}}+H . c .,
$$

where, $\mathscr{O}_{i}^{(6)}$ and $c_{i}^{(6)}$ represent gauge invariant local operators and dimensionless Wilson coefficients, respectively. In the Higgs sector, the effective operators include the anomalous Higgs-gauge boson interactions [3]. Up to the first power of Higgs particle, the effective Lagrangian can be written in terms of the physical fields as $\mathscr{L}_{\text {eff. }}^{(6)}=H Z_{\mu} T^{\mu v} A_{v}+H . c$., and the $T^{\mu v}$ vertex of the $H Z \gamma$ interaction in momentum space is $[8,9]$

$$
T^{\mu v}(k, Q)=\hat{\alpha}(k, Q) Q^{2} g^{\mu v}+\alpha_{1}(k, Q)\left[Q \cdot k g^{\mu v}-Q^{\mu} k^{v}\right]+\alpha_{2}(k, Q) \varepsilon^{\mu v \rho \sigma} Q_{\rho} k_{\sigma} .
$$

Here, $k$ and $Q$ denote the $Z$ boson and photon momenta and $\varepsilon_{\mu v \rho \sigma}$ is a totally antisymmetric tensor with $\varepsilon_{0123}=1$. Practically, $\left(\hat{\alpha}, \alpha_{1}, \alpha_{2}\right)$ are dimensionful independent coefficients whose nonzero values are possibly induced by non-SM heavy particles and can change Higgs production cross sections. In the SM the leading order results are $\hat{\alpha}^{\mathrm{SM}}=\alpha_{1}^{\mathrm{SM}}=\alpha_{2}^{\mathrm{SM}}=0$. In this notation, $\hat{\alpha}, \alpha_{1}$ are the coefficients of the CP-even operators and $\alpha_{2}$ is the coupling in the CP-odd interaction term. 
These couplings depend on gauge boson masses, the $S U(2)_{\mathrm{L}}$ coupling constant, $g$, the weak mixing angle, $\theta_{\mathrm{w}}$, and should also be constrained in searching for NP effects [10].

The Feynman diagram of the Higgs production subprocess $\gamma q \rightarrow \gamma Z q \rightarrow H q$ at leading order, is illustrated in Fig. 1. In the forward collisions, the emitted photons are commonly considered

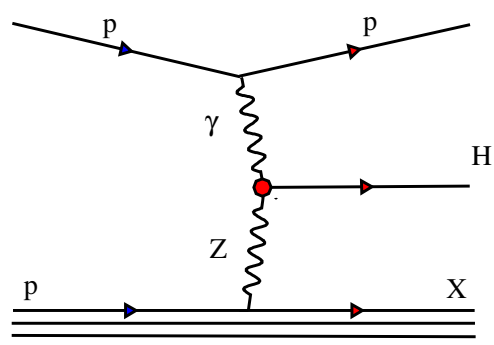

Figure 1: The Feynman diagram of the subprocess $\gamma q \rightarrow \gamma Z q \rightarrow H q$ at leading order.

massless particles in the equivalent photon approximation (EPA) method [11]. In the EPA the photon spectrum is given by

$$
f\left(E_{\gamma}, Q^{2}\right)=\frac{d N}{d E_{\gamma} d Q^{2}}=\frac{\alpha_{\mathrm{e}}}{\pi} \frac{1}{E_{\gamma} Q^{2}}\left[\left(1-\frac{E_{\gamma}}{E_{\mathrm{p}}}\right)\left(1-\frac{Q_{\min }^{2}}{Q^{2}}\right) F_{\mathrm{E}}+\frac{E_{\gamma}^{2}}{2 E_{\mathrm{p}}^{2}} F_{\mathrm{M}}\right], \quad \alpha_{\mathrm{e}}=\frac{e^{2}}{4 \pi},
$$

where, $F_{\mathrm{M}}$ and $F_{\mathrm{E}}$ are proton magnetic and electric form factors, respectively [12],

$$
\begin{array}{llll}
F_{\mathrm{E}}=\frac{4 m_{\mathrm{p}}^{2} G_{\mathrm{E}}^{2}+Q^{2} G_{\mathrm{M}}^{2}}{4 m_{\mathrm{p}}^{2}+Q^{2}}, & G_{\mathrm{E}}^{2}=\frac{G_{\mathrm{M}}^{2}}{\mu_{\mathrm{p}}^{2}}=\left(1+\frac{Q^{2}}{Q_{0}^{2}}\right)^{-4}, & F_{\mathrm{M}}=G_{\mathrm{M}}^{2}, & \mu_{\mathrm{p}}^{2}=7.78, \\
Q_{\text {min }}^{2}=\frac{E_{\gamma}^{2} m_{\mathrm{p}}^{2}}{E_{\mathrm{p}}\left(E_{\mathrm{p}}-E_{\gamma}\right)}, & Q_{0}^{2}=0.71 G e V^{2}, & E_{\gamma}=E_{\mathrm{p}} \xi, & m_{\mathrm{p}}=0.938 \mathrm{GeV} .
\end{array}
$$

In the following, due to the $Q^{2}=0$ approximation the $\hat{\alpha}$ coupling disappears in the scattering amplitude relation. For hard scattering matrix elements, we use the CTEQ14 collaboration results in leading order for the parton distribution functions (PDFs) [13]. The uncertainties due to the PDF choice are estimated as $0.022 \%, 0.019 \%$, and $0.161 \%$ for the first, second, and third acceptance regions at $\sqrt{s}=14 \mathrm{TeV}$, respectively [14]. Integrating the product of the subprocess cross section, the photon spectrum from Eq. (1.3), and also a selected PDF set leads to the total cross section in which the integration limits are determined by corresponding conservation laws. Using

$$
\begin{array}{ll}
\mathrm{y}_{\min }=\operatorname{Max}\left[\frac{\omega^{2}}{4 E_{\mathrm{p}} x_{\max }}, E_{\mathrm{p}} \xi_{\min }\right], & \mathrm{y}_{\max }=\operatorname{Min}\left[\frac{\omega^{2}}{4 E_{\mathrm{p}} x_{\min }}, E_{\mathrm{p}} \xi_{\max }\right], \\
\omega_{\min }=\operatorname{Max}\left[2 E_{\mathrm{p}} \sqrt{\xi_{\min } x_{\min }}, m_{\mathrm{H}}+m_{\mathrm{q}}\right], & \omega_{\max }=2 E_{\mathrm{p}} \sqrt{\xi_{\text {max }} x_{\max }},
\end{array}
$$

the total cross section is derived

$$
\sigma=\sum_{q=u, d, s, c, b} \int_{\omega_{\min }}^{\omega_{\max }} \frac{\omega}{2 E_{\mathrm{p}} y} d \omega \int_{y_{\min }}^{y_{\max }} d y \int_{Q_{1, \min }^{2}}^{Q_{\max }^{2}} d Q_{1}^{2} f_{\gamma}\left(y, Q_{1}^{2}\right) f_{\mathrm{q}}\left(\frac{\omega^{2}}{4 E_{\mathrm{p}} y}, Q_{2}^{2}\right) \hat{\sigma}_{Z \gamma \rightarrow H}\left(Q_{1}^{2}, \omega, y\right) .
$$


Numerical results of relation (1.6) show that there is no considerable difference between the couplings $\alpha_{1}$ and $\alpha_{2}$. In this paper, the factorization scale, $\mu_{\mathrm{f}}$, the renormalization scale, $\mu_{\mathrm{r}}$, and the threshold production scale, $Q_{2}$, are all considered equal to the Higgs mass, $m_{\mathrm{H}}=125 \mathrm{GeV}$.

\section{Constraints on the Higgs-Gauge Boson Anomalous Couplings}

To perform the numerical analysis on the couplings constraints from the process $p p \rightarrow p \gamma p \rightarrow$ $p H X$ at the LHC, we need the expected background and signal events in the relevant Higgs decay channels. The combined results of three channels $H \rightarrow \gamma \gamma, H \rightarrow W^{+} W^{-}$, and $H \rightarrow Z Z$ are discussed and the SM branching ratios for these decay channels are $2.28 \times 10^{-3}, 2.15 \times 10^{-1}$, and $2.64 \times 10^{-2}$, respectively [15]. The number of signal events for each final state, $N_{\text {signal }}$, at a specific integrated luminosity, $\mathscr{L}_{\text {int }}$, is theoretically given by

$$
N_{\text {signal }}\left(\alpha_{1}, \alpha_{2}\right)=\sigma(p p \rightarrow p H X) \times B r(H \rightarrow F F) \times B r\left(F \rightarrow f_{1} f_{2} \ldots\right) \times \mathscr{L}_{\text {int }} .
$$

Here, $F=\gamma, W^{ \pm}, Z$, and $f=l^{ \pm}, v_{l}$ (for $F=W, Z$ ), also $\operatorname{Br}\left(W \rightarrow f_{1} f_{2} \ldots\right)=0.05$, and $\operatorname{Br}(Z \rightarrow$ $\left.f_{1} f_{2} \ldots\right)=0.12$. We take into account the irreducible background, $(\gamma+q \rightarrow H+q)$, in diffractive processes as well as the reducible ones. As we found, the contribution of the reducible photoproduction processes is rejected by applying the cuts and is more smaller than that of the irreducible process. The background cross sections, calculated with CompHEP v4.5.2 package are in Ref. [16]. The final state includes an intact proton in addition to $\gamma \gamma+$ jet, $l_{1}^{ \pm} l_{2}^{\mp} v_{l_{1}} v_{l_{2}}+$ jets, and $l_{1}^{ \pm} l_{1}^{\mp} l_{2}^{ \pm} l_{2}^{\mp}$, for $\gamma \gamma, W^{+} W^{-}$, and $Z Z$ decay channels, respectively. This state is considered to calculate the background subprocesses. In this analysis, we use the survival factor, $\varepsilon=0.74$, which depends on the detector performance, and at the scale of Higgs mass results in a $\sim 26 \%$ reduction of the expected signal and background cross sections [17]. Moreover, the reconstruction and acceptance efficiencies are not included into the bounds estimations in this paper.

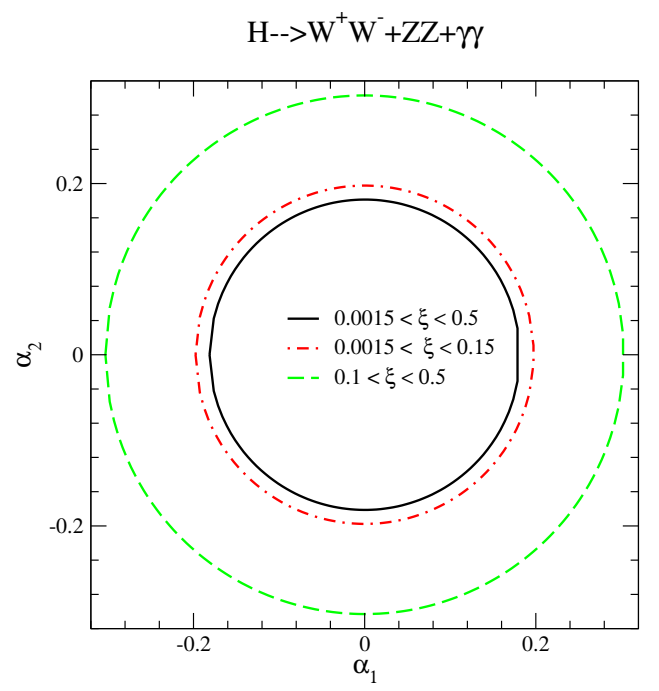

Figure 2: The contour diagrams in $\alpha_{2}-\alpha_{1}$ plane (units in $\mathrm{TeV}^{-1}$ ) for the combined channel of three different Higgs decay channels with 95\% C.L. at $\sqrt{s}=14 \mathrm{TeV}, \varepsilon=0.74$, and for $\mathscr{L}_{\text {int }}=300 \mathrm{fb}^{-1}$. The curves are plotted for three different acceptance regions. 
The contour diagrams of the bounds in the combined channel are studied in Fig. 2, in $\alpha_{2}-\alpha_{1}$ plane, with $95 \%$ C.L. at $\sqrt{s}=14 \mathrm{TeV}, \varepsilon=0.74$, and for $\mathscr{L}_{\text {int }}=300 \mathrm{fb}^{-1}$ in three $\xi$ values. As is shown in Fig. 2, the first acceptance region is the most sensitive interval of $\xi$ to the anomalous couplings and this point could be estimated by a counting experiment analysis. Figs. 3 and 4 display the dependencies of $\alpha_{i=1,2}$ to the integrated luminosity and survival factor, respectively. The curves are depicted at $\sqrt{s}=14 \mathrm{TeV}$ and for three different acceptance regions. Increasing

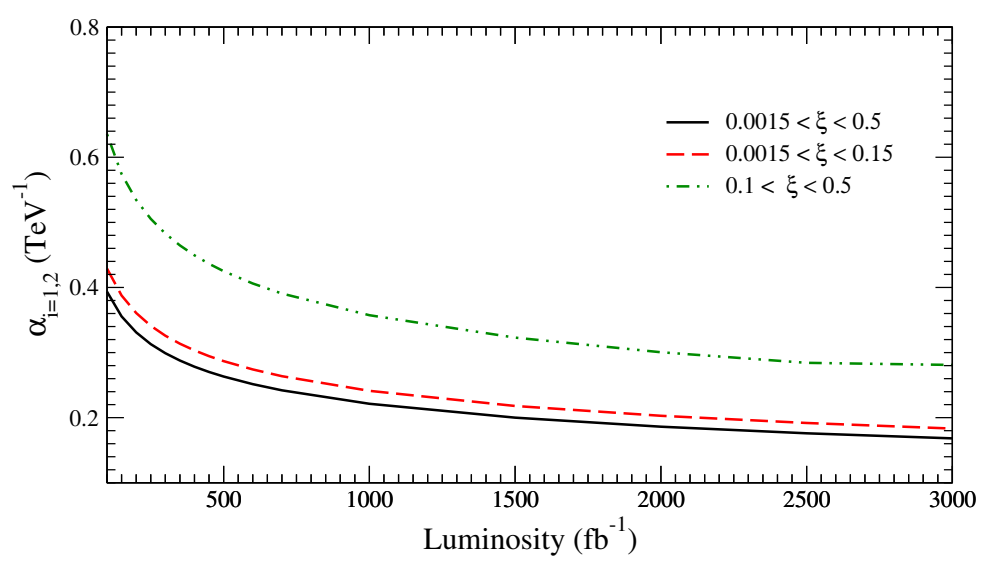

Figure 3: The anomalous couplings as a function of integrated luminosity, at $\sqrt{s}=14 \mathrm{TeV}$ and $\varepsilon=0.74$. The curves are plotted for three different acceptance regions.

the luminosity as well as survival factor provide more restricted bounds in all acceptance regions. To have more realistic constraints, the appropriate cuts, which select the events, are applied on pseudorapidities and transverse momenta of the final state particles. Moreover, some mass cuts to suppress the background events at each decay channel are imposed.

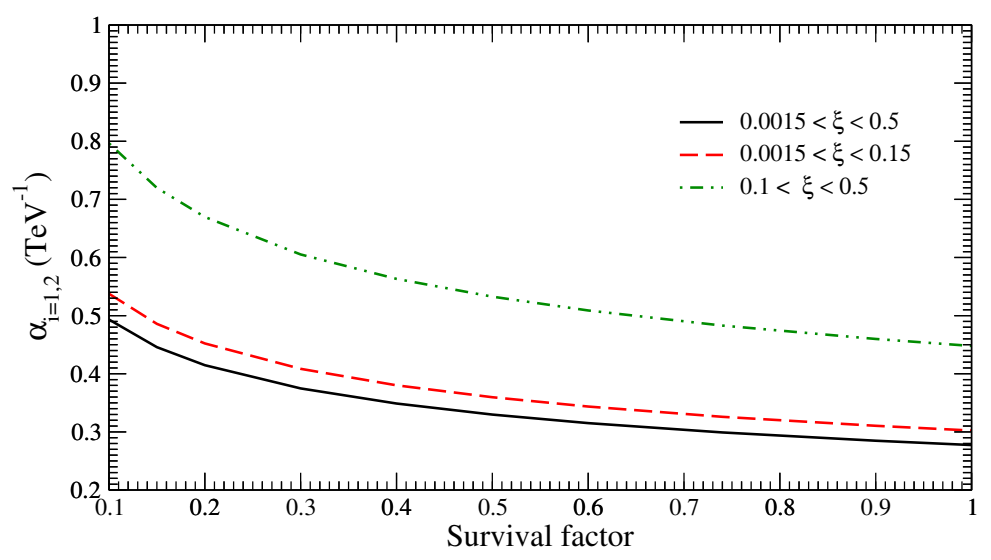

Figure 4: The anomalous couplings as a function of survival factor, at $\sqrt{s}=14 \mathrm{TeV}$ and $\mathscr{L}_{\text {int }}=300 \mathrm{fb}^{-1}$. The curves are plotted for three different acceptance regions.

The SM loop computation at $m_{\mathrm{H}}=125 \mathrm{GeV}$, ignoring bottom quark contributions, predicts a coupling value of about $\alpha_{1}=-4.1 \times 10^{-5} \mathrm{GeV}^{-1}$ [3]. According to the CMS (ATLAS) measurements at center of mass energy $\sqrt{s}=8 \mathrm{TeV}$ and the luminosity $\mathscr{L}_{\text {int }}=19.6 \mathrm{fb}^{-1}$, the constraint on the anomalous coupling is $-0.162 \leq \alpha_{1} \leq 0.082 \mathrm{TeV}^{-1}\left(-0.168 \leq \alpha_{1} \leq 0.088 \mathrm{TeV}^{-1}\right)$ [18] 
([19]). Comparing the results of Figs. 2-4 with the corresponding ones reported in Ref. [3], $\left|\alpha_{1}\right| \leq 2$ $\mathrm{TeV}^{-1}$, one can find that our proposed channel is more sensitive to probe the $H Z \gamma$ couplings. Following additional processes to suppress backgrounds with a real analysis on experimental data can help to improve the constraints that phenomenologically extracted here. In the present paper, we can emphasize that detecting particles in the forward regions is a reasonable method to explore the anomalous $H Z \gamma$ vertices. We conclude that besides the other channels, the studied Higgs photoproduction process is complementary to search for the NP effects at the LHC future run.

\section{ACKNOWLEDGMENTS}

This work was supported in parts by Islamic Azad University Central Tehran Branch. I would also like to thank the organizers of DIS2016 for their warm hospitality and financial support.

\section{References}

[1] H. Belusca-Maito, arXiv:1507.05657 [hep-ph].

[2] A. Falkowski, arXiv:1505.00046 [hep-ph]. C. Englert, R. Kogler, H. Schulz and M. Spannowsky, arXiv:1511.05170 [hep-ph]. T. Han, Y. P. Kuang and B. Zhang, Phys. Rev. D 73, 055010 (2006) [hep-ph/0512193].

[3] E. Masso and V. Sanz, Phys. Rev. D 87, no. 3, 033001 (2013) [arXiv:1211.1320 [hep-ph]].

[4] S. Chatrchyan et al. [CMS Collaboration], Phys. Lett. B 726 (2013) 587 [arXiv:1307.5515 [hep-ex]].

[5] G. Aad et al. [ATLAS Collaboration], Phys. Lett. B 732, 8 (2014) [arXiv:1402.3051 [hep-ex]].

[6] T. Corbett, O. J. P. Eboli, J. Gonzalez-Fraile and M. C. Gonzalez-Garcia, Phys. Rev. D 87, 015022 (2013) [arXiv:1211.4580 [hep-ph]].

[7] M. G. Albrow et al. [FP420 R and D Collaboration], JINST 4, T10001 (2009) [arXiv:0806.0302 [hep-ex]].

[8] V. Hankele, G. Klamke, D. Zeppenfeld and T. Figy, Phys. Rev. D 74, 095001 (2006) [hep-ph/0609075].

[9] K. Hagiwara, S. Ishihara, R. Szalapski and D. Zeppenfeld, Phys. Rev. D 48, 2182 (1993).

[10] P. Achard et al. [L3 Collaboration], Phys. Lett. B 589, 89 (2004) [hep-ex/0403037].

[11] G. Baur, K. Hencken, D. Trautmann, S. Sadovsky and Y. Kharlov, colliders,” Phys. Rept. 364, 359 (2002) [hep-ph/0112211].

[12] V. M. Budnev, I. F. Ginzburg, G. V. Meledin and V. G. Serbo, Phys. Rept. 15, 181 (1975).

[13] S. Dulat et al., Phys. Rev. D 93, no. 3, 033006 (2016) [arXiv:1506.07443 [hep-ph]].

[14] J. Butterworth et al., J. Phys. G 43, 023001 (2016) [arXiv:1510.03865 [hep-ph]].

[15] S. Dittmaier et al., arXiv:1201.3084 [hep-ph].

[16] A. Pukhov et al., hep-ph/9908288.

[17] L. A. Harland-Lang, V. A. Khoze and M. G. Ryskin, Eur. Phys. J. C 76, no. 5 , 255 (2016) [arXiv:1601.03772 [hep-ph]].

[18] [CMS Collaboration], arXiv:1307.7135.

[19] ATLAS Collaboration, ATL-PHYS-PUB-2013-014, https://cds.cern.ch/record/1611186, (2013). 